\title{
Operative hysteroscopy-assisted pregnancy termination after failed surgical abortion in missed abortion of woman with complete septate uterus
}

\author{
Soomin Ryu, MD ${ }^{1}$, Hye Won Baek, MD¹, Inha Lee, MD ${ }^{1,2}$, Young Bin Won, MD ${ }^{1,2}$, Heeyon Kim, MD ${ }^{1,3}$, \\ Jae Hoon Lee, MD ${ }^{1,2}$, Bo Hyon Yun, MD,PhD ${ }^{1,2}$, Joo Hyun Park, MD, PhD ${ }^{1,3}$, Seok Kyo Seo, MD, PhD ${ }^{1,2}$, \\ SiHyun Cho, MD, PhD ${ }^{1,3}$, Young Sik Choi, MD, PhD ${ }^{1,2}$, Byung Seok Lee, MD, PhD ${ }^{1,2}$

\footnotetext{
${ }^{1}$ Department of Obstetrics and Gynecology, Severance Hospital, ${ }^{2}$ Institute of Women's Life Medical Science, ${ }^{3}$ Department of Obstetrics and Gynecology, Gangnam Severance Hospital, Yonsei University College of Medicine, Seoul, Korea
}

First trimester surgical abortion is an effective and safe procedure. Although its failure is uncommon, congenital uterine anomaly may be considered as one of the etiologic factors in such cases. Here, we report a rare case of surgical abortion failure that was successfully managed by operative hysteroscopy-assisted dilatation and evacuation (D\&E) under ultrasound guidance in a woman with complete uterine septum. The patient was referred to Severance Hospital after two consecutive surgical abortion failures even under ultrasound guidance. A missed abortion in a leftsided hemicavity of septate uterus was noted on ultrasonography. Ultrasound-guided D\&E was unsuccessful because the curette could not reach the uterine cavity with the gestational sac. Operative hysteroscopy revealed insufficient communication with the left-sided cavity just above the cervical internal os of the uterine septum. After widening the communication, ultrasound-guided D\&E was successfully performed.

Keywords: Uterine anomalies; Septate uterus; Missed abortion; Induced abortion; Hysteroscopy

\section{Introduction}

Congenital malformations of the female genital tract are defined as deviations from the normal anatomy that result from embryological maldevelopment of the Müllerian or paramesonephric ducts $[1,2]$. Septate uterus is the most common congenital uterine anomaly resulting from the failure of resorption of the midline septum after the fusion of two Müllerian ducts. Septate uterus is defined as a uterus with a normal outline and an internal indentation at the fundal midline exceeding $50 \%$ of the uterine wall thickness. Septate uteri are subdivided into two sub-classes according to the degree of uterine corpus deformity: incomplete/partial septate and complete septate uterus. A partial septate uterus is a single fundus and cervix with a uterine septum that partially divides the uterine cavity above the internal cervical os, and a complete septate uterus is characterized by the existence of a septum that fully divides the uterine cavity up to the internal cervical os with or without a cervical and/or vaginal septum [1]. Adverse reproductive outcomes that have been attributed to septate uteri include infertility, pregnancy loss, and poor obstetric outcomes, such as malpresentation and preterm delivery. However, many women with uterine septa do not experience any reproductive difficulties $[2,3]$.

First trimester surgical abortion is an effective and safe procedure when its completion can be confirmed with careful inspection of the aspirate [4]. However, if attempts at surgical abortion fail, steps must be taken to carefully assess the

Received: 2019.05.28. Revised: 2019.08.08. Accepted: 2019.10.10. Corresponding author: Young Sik Choi, MD, PhD

Department of Obstetrics and Gynecology, Yonsei University College of Medicine, 50-1 Yonsei-ro, Seodaemun-gu, Seoul 03722, Korea

E-mail: yschoi08@yuhs.ac

https://orcid.org/0000-0002-1157-4822

Articles published in Obstet Gynecol Sci are open-access, distributed under the terms of the Creative Commons Attribution Non-Commercial License (http://creativecommons. org/licenses/by-nc/3.0/) which permits unrestricted non-commercial use, distribution, and reproduction in any medium, provided the original work is properly cited.

Copyright $\odot 2020$ Korean Society of Obstetrics and Gynecology 


\section{Obstetrics \& Gynecology Science}

Soomin Ryu, et al. Surgical abortion in septate uterus

clinical technique and patient's anatomy. Uterine anomaly has been recognized as an occasional contributing factor to failed surgical abortion [5].

Here, we present a case that was successfully managed with operative hysteroscopy-assisted dilatation and evacuation (D\&E) under ultrasound guidance in a woman with complete septate uterus in whom previous ultrasound-guided D\&E for the management of missed abortion had failed.

\section{Case report}

A 33-year-old woman (gravida 2 para 1) with a history of amenorrhea for 8 weeks and 4 days presented with two consecutive failed surgical abortions. She had a previous history of preterm delivery by cesarean section at gestational age of 33 weeks under the diagnosis of breech presentation in 2011. A uterine anomaly was found incidentally during surgery. On May 3, 2014, pregnancy was confirmed by detecting gestational sac in the endometrial cavity using ultrasonography at 5 weeks and 2 days of gestation. One week later, the crown-lump length (CRL) was consistent with an age of $6+0$ weeks without a fetal heartbeat. At 8 weeks of gestation, a fetal heartbeat was still not observed and CRL had not increased. Under the diagnosis of missed abortion, she planned to undergo D\&E. Ultrasound-guided D\&E was attempted, but the suction cannula could not reach the uterine cavity with the gestational sac at a local clinic and a secondary referral hospital.

After the failed surgical abortion, she was referred to our hospital. A pelvic examination revealed a single uterine cervical opening without vaginal septum. Ultrasonography revealed two separate uterine cavities with a $2.8-\mathrm{cm}$ gesta- tional sac in the left-sided cavity but no definite fetal pole. A septum dividing the uterine cavity was observed, but fundal indentation was not definite (Fig. 1A). For pregnancy termination, ultrasound- and/or operative hysteroscopyguided surgical abortion was planned. The day before the operation, misoprostol $400 \mu \mathrm{g}$ was inserted intravaginally to soften the uterine cervix. After adequate dilatation of the uterine cervix, a curette was inserted into the uterine cavity under ultrasound guidance; however, it did not reach the left-sided uterine cavity containing the gestational sac. When a hysteroscope was inserted into the uterine cavity, it passed easily into the empty right-sided uterine cavity but could not be passed into the left-sided cavity. During the operation, slight communication to left-sided cavity was noted just above the cervical internal os. After the communication was widened using hysteroscopic resection of the uterine septum, the hysteroscope could enter the left-sided cavity with the conceptual tissue (Fig. 1B-D). After further resection of the uterine septum, conceptual tissue was successfully removed by curettage and vacuum aspiration under ultrasound guidance. Because the placental tissue was within the uterine septum, we did not perform a septectomy. The patient was discharged without complications on the same day. Two weeks later, magnetic resonance imaging (MRI) was performed to enable a more accurate diagnosis of the Müllerian anomaly. MRI revealed that the septum extended nearly to the external cervical os without fundal indentation, suggesting complete septate uterus with cervical septum. There were no specific findings in the endometrial cavity or either ovary. There was no urologic anomaly (Fig. 2A). According to the European Society for Human Reproduction and Embryology/European Society of Gynecological Endoscopy (ESHRE/ ESGE) classification system, the female genital anomaly was
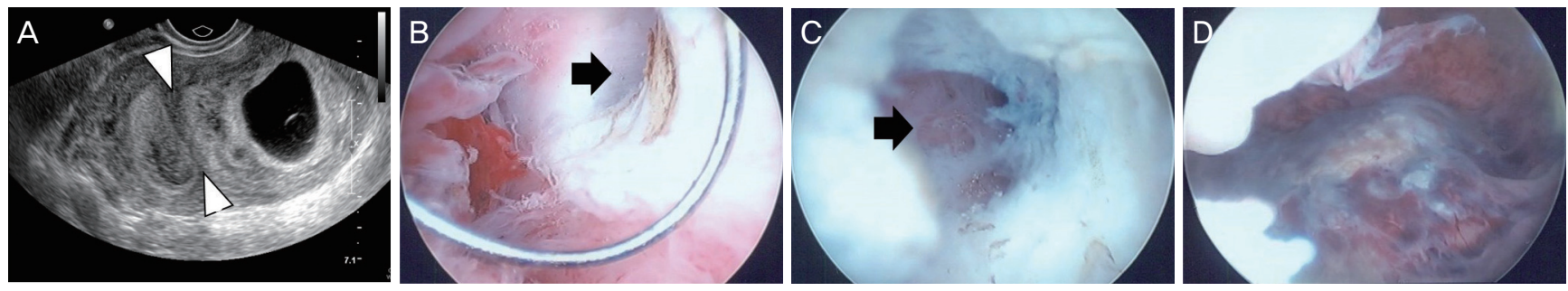

Fig. 1. Ultrasonographic finding $(A)$ and operative findings during hysteroscopy for dilatation and evacuation (B-D). (A) Transverse scan showing two uterine cavities with a gestational sac in the left-sided hemicavity, (B) tiny septal communication between the two hemicavities, (C) further widened communication using hysteroscopy, and (D) gestational tissues in the left hemicavity (arrowhead, uterine septum; arrow, communication between right and left hemicavities). 


\section{Obstetrics \& Gynecology Science}

Vol. 63, No. 1, 2020

classified as U2bC1V0.

Because the patient experienced adverse obstetrical events, including preterm delivery and miscarriage, an operative hysteroscopic septectomy was planned for the next pregnancy. Upon entry to the endometrial cavity, diffuse endometrial polyps and a uterine septum were noted (Fig. 2B). However, a cervical septum was not found. Operative hysteroscopic uterine septectomy and endometrial polypectomy were performed on September 5, 2014. She was discharged uneventfully later the same day. After the operation, an intrauterine Foley catheter was inserted for 1 week, and high-dose continuous cyclic estrogen-progestin therapy with conjugated equine estrogen $1.25 \mathrm{mg}$ was taken PO bid for 28 days, followed by medroxyprogesterone acetate $10 \mathrm{mg}$ qd for 14 days for 2 months. The pathology finding of the uterine septum was "endomyometrial tissue showing proliferative phase."

\section{Discussion}

Surgical abortion is a very safe procedure when performed legally. The overall major complication rate for first trimester surgical abortions is about $0.5 \%$. Complications include failed abortion, incomplete abortion, hemorrhage, infection, and uterine perforation [6]. Although failed abortion is very rare, with an incidence of $0.08-4.2 \%$, it may have serious consequences such as bleeding, infection, need for addition- al surgery, and persistent pregnancy. Causes of failed abortion include poor technique, cervical stenosis, marked uterine anteversion or retroversion, endometrial distortion, uterine fibroids, very early gestational age, and uterine anomaly [7]. There are several reports on failed surgical termination in patients with uterine anomalies, including bicornuate uteri [8], non-communicating rudimentary horn $[9,10]$, and septate uteri $[7,11]$. Failed surgical abortion was also reported in women with Robert's uterus, a rare variant of septate uterus consisting of a non-communicating hemicavity or a contralateral unicornuate uterine cavity in a single uterine body with a normal fundus [12]. In Korea, however, there are few reports on failed surgical abortion in patients with uterine anomalies. In fact, we found only one case report on hysteroscopyassisted therapeutic abortion after two consecutive failures of induced abortion in a woman with uterus didelphys, obstructed hemivagina, and cervicovaginal fistula [13]. Here, we reported a rare case that was successfully managed with operative hysteroscopy-assisted D\&E under ultrasound guidance in a woman with a complete septate uterus in whom ultrasound-guided D\&E had failed to manage a missed abortion.

Although ultrasound guidance is not routinely used during surgical abortion, it may increase the success rate and decrease the incidence of retained products of conception and uterine perforation. Ultrasound-guided surgical abortion is more valuable in cases of surgical abortion in women with uterine anomaly as well as after failed abortion [7,11].
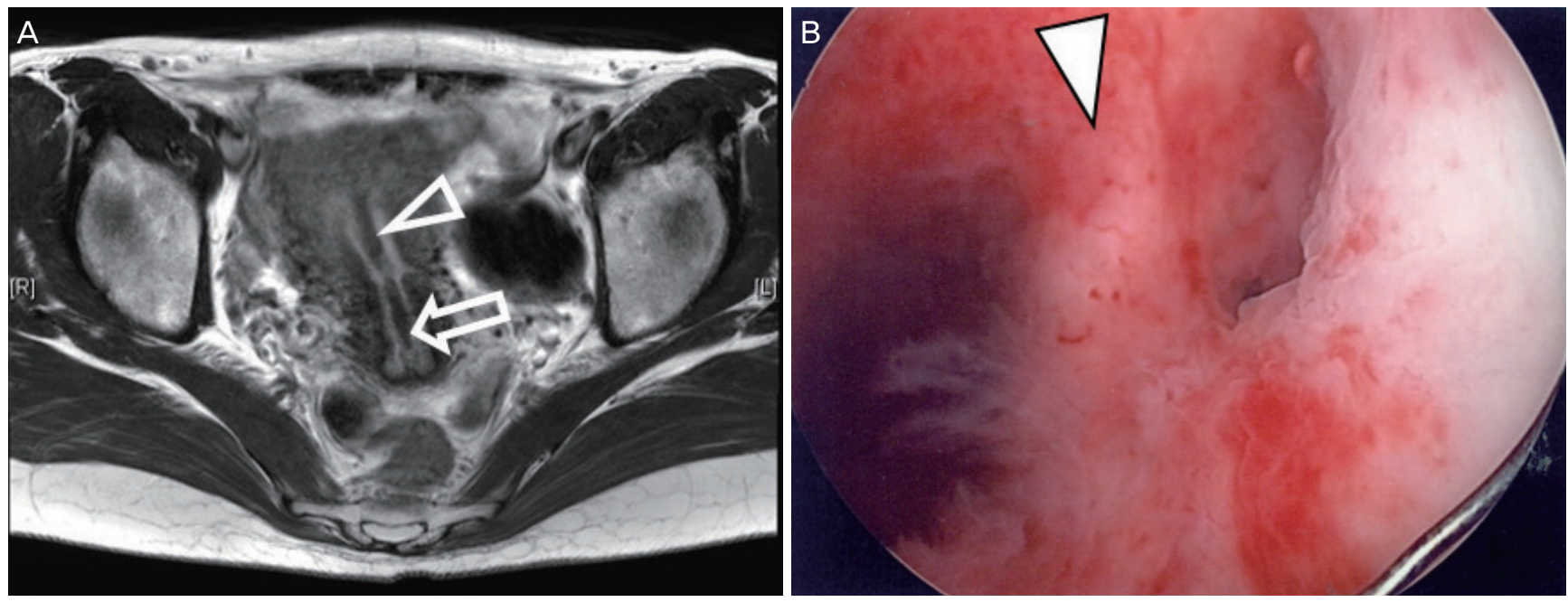

Fig. 2. T2-weighted magnetic resonance imaging ( $A$ ) and operative findings during hysteroscopy for septectomy (B) (arrowhead, uterine septum; arrow, cervical septum). 


\section{Obstetrics \& Gynecology Science}

Soomin Ryu, et al. Surgical abortion in septate uterus

However, ultrasound guidance may not be useful in cases of uterine anomaly with no or small communication. In the present case, a suction cannula could not reach the left-sided pregnant hemicavity despite ultrasound guidance.

Hysteroscopy can be useful in identifying pregnancy in women with a uterine anomaly. This is most helpful in the case of septate uterus $[7,11]$. In the present study, MRI revealed that the patient had complete septate uterus with cervical septum, but the left cervical canal and cervical septum could not be identified upon entering the cervix using a hysteroscope after uterine cervical dilatation. We identified the tiny communication just above isthmus level and a leftsided hemicavity with conceptual tissue. After widening the communication, we easily performed D\&E under ultrasound guidance.

In the present case, a female genital anomaly was classified into U2bC1VO according to the ESHRE/ESGE classification system. Unlike on MRI, however, the cervical septum could not be identified during hysteroscopy. Because the right-sided cervical canal was predominant and the left-sided cervical canal was too narrow to pass through, the right-sided canal was passed easily after cervical dilatation. The genital anomaly might have been Robert's uterus with an inactive blind hemicavity without hematometra. Communication of the isthmic portion might have been created during the previous Cesarean section. It was previously suggested that fusion and resorption begin at the isthmus and proceed simultaneously in the cranial and caudal directions [14]. Although fusion and resorption defects frequently affect the superior uterine segment only or both segments with the transversal limit of the junction between them at an isthmic level (corresponding to the second portion or fusion area of both Müllerian ducts), segmental defects are possible [15]. Although it has not been reported until now, Robert's uterus with isthmic communication may be possible.

In conclusion, women with female genital anomaly are at risk of failed surgical abortion. In such patients, operative hysteroscopy and ultrasound are useful for locating the site of pregnancy and successfully performing the surgical abortion.

\section{Acknowledgments}

This study was supported by a faculty research grant from
Yonsei University College of Medicine (6-2017-0189).

\section{Conflict of interest}

No potential conflict of interest relevant to this article was reported.

\section{Ethical approval}

The study was approved by the Institutional Review Board of Severance Hospital (4-2019-0268) and performed in accordance with the principles of the Declaration of Helsinki.

\section{Patient consent}

The Institutional Review Board waived the need for patient consent under the restriction that no identifiable personal information is revealed in the process.

\section{References}

1. Grimbizis GF, Gordts S, Di Spiezio Sardo A, Brucker S, De Angelis C, Gergolet M, et al. The ESHRE/ESGE consensus on the classification of female genital tract congenital anomalies. Hum Reprod 2013;28:2032-44.

2. Practice Committee of the American Society for Reproductive Medicine. Uterine septum: a guideline. Fertil Steril 2016;106:530-40.

3. Valle RF, Ekpo GE. Hysteroscopic metroplasty for the septate uterus: review and meta-analysis. J Minim Invasive Gynecol 2013;20:22-42.

4. Tristan SB, Gilliam M. First trimester surgical abortion. Clin Obstet Gynecol 2009;52:151-9.

5. Goldthwaite LM, Teal SB. Controversies in family planning: pregnancy termination in women with uterine anatomic abnormalities. Contraception 2014;90:460-3.

6. Hakim-Elahi E, Tovell HM, Burnhill MS. Complications of first-trimester abortion: a report of 170,000 cases. Obstet Gynecol 1990;76:129-35.

7. Pennes DR, Bowerman RA, Silver TM, Smith SJ. Failed first trimester pregnancy termination: uterine anomaly 


\section{Obstetrics \& Gynecology Science}

Vol. 63, No. 1, 2020

as etiologic factor. J Clin Ultrasound 1987;15:165-70.

8. Lazenby GB, Huang C, Rahall AM, Fogelson NS. Pregnancy termination via laparotomy in a woman with bicornuate uterus. Contraception 2007;75:241-3.

9. Cutner A, Saridogan E, Hart R, Pandya P, Creighton S. Laparoscopic management of pregnancies occurring in non-communicating accessory uterine horns. Eur J Obstet Gynecol Reprod Biol 2004;113:106-9.

10. Soundararajan $V$, Rai J. Laparoscopic removal of a rudimentary uterine horn during pregnancy. A case report. J Reprod Med 2000;45:599-602.

11. Valle RF, Sabbagha RE. Management of first trimester pregnancy termination failures. Obstet Gynecol 1980;55:625-9.

12. Singhal $S$, Agarwal $U$, Sharma D, Sirohiwal D. Pregnancy in asymmetric blind hemicavity of Robert's uterus--a previously unreported phenomenon. Eur J Obstet Gynecol Reprod Biol 2003;107:93-5.

13. Roh JR, Choi DS, Kim BH, Seo YS, Lee JH. A case of hysteroscopy assisted therapeutic abortion of pregnancy in a side of uterus didelphys affected with obstructed hemivagina. Korean J Obstet Gynecol 1998;41:1972-5.

14. Muller P, Musset R, Netter A, Solal R, Vinourd JC, Gillet JY. State of the upper urinary tract in patients with uterine malformations. Study of 133 cases. Presse Med 1967;75:1331-6.

15. Acién $P$, Acién $M$, Sánchez-Ferrer ML. Müllerian anomalies "without a classification": from the didelphysunicollis uterus to the bicervical uterus with or without septate vagina. Fertil Steril 2009;91:2369-75. 